\title{
Neuromorphic behavior in percolating nanoparticle films
}

\author{
Shawn Fostner and Simon A. Brown* \\ The MacDiarmid Institute for Advanced Materials and Nanotechnology, University of Canterbury, Private Bag 4800, \\ Christchurch 8140, New Zealand \\ and Department of Physics and Astronomy, University of Canterbury, Private Bag 4800, Christchurch 8140, New Zealand
}

(Received 2 August 2015; published 23 November 2015)

\begin{abstract}
We show that the complex connectivity of percolating networks of nanoparticles provides a natural solid-state system in which bottom-up assembly provides a route to realization of neuromorphic behavior. Below the percolation threshold the networks comprise groups of particles separated by tunnel gaps; an applied voltage causes atomic scale wires to form in the gaps, and we show that the avalanche of switching events that occurs is similar to potentiation in biological neural systems. We characterize the level of potentiation in the percolating system as a function of the surface coverage of nanoparticles and other experimentally relevant variables, and compare our results with those from biological systems. The complex percolating structure and the electric field driven switching mechanism provide several potential advantages in comparison to previously reported solid-state neuromorphic systems.
\end{abstract}

DOI: 10.1103/PhysRevE.92.052134

PACS number(s): 64.60.ah, 73.63.-b, 36.40.-c, 73.23.Hk

\section{INTRODUCTION}

The experimental realization [1] of memristors [2] and atomic switches [3] has recently led to proposals for novel computational paradigms [4,5], and in particular to proposals for architectures that would enable neuromorphic computing $[6,7]$ using complex networks of nanoscale devices. A key to such proposals is that the behavior of individual component devices is dependent on the history of their inputs (i.e., their state exhibits "memory"), and hence that the behavior of such devices can be seen as analogous to that of the synapses between neurons, which enable learning in the human brain [8-14]. In particular, it has been shown that nanoscale devices can exhibit both short-term plasticity (STP) and long-term potentiation (LTP) $[8,11]$.

Memristors have been identified as the fourth basic circuit element (alongside resistors, capacitors, and inductors) [1,2]. The first memristive devices were based on movement of impurities in semiconducting matrices [1], and the first atomic switches relied on the electrochemical reduction of $\mathrm{Ag}_{2} \mathrm{~S}$ to form atomic scale silver wires [3]. Since then, many other related devices have been reported, including those based on, for example, polymers [15], indium zinc oxide [16], and superconductors [17].

Neuromorphic behavior is a property of a system that comprises many such devices. There have been a variety of proposals for both deterministically assembled and selfassembled systems that exhibit neuromorphic behavior in some form, with some impressive results achieved [6,7,16,1822]. The considerable current excitement about neuromorphic behavior is driven at least partially by the possibility that that the collective behavior of these system will exhibit emergent behavior similar to that which occurs in the brain [23]. Other drivers include the belief that neuromorphic behavior may enable new forms of computational capability, such as reservoir computing [7,24,25], and the hope that these electronic systems will allow a deeper understanding of the fundamental nature of intelligence $[5,20,23,26]$. One of the

\footnotetext{
*simon.brown@canterbury.ac.nz
}

key motivations for the present work is that the percolating system of interest is poised near criticality, as are biological neural systems [23].

We have recently [27] reported switching behavior (along with quantized conduction [28-30] at room temperature) in devices composed of tin nanoparticles. In this system the random deposition of the nanoparticles leads to formation of a complex percolating [31] network comprising groups of particles separated by tunnel gaps. When a voltage is applied across the system the local electric field across the tunnel gaps can be very high resulting in switching events driven by the formation of atomic scale wires in the gaps [27]. Here we model electrical transport across these devices and demonstrate that this complex system fulfills at least some of the criteria for neuromorphic behavior. Previous simulations of neuromorphic behavior focused on harmonic generation in quite different physical systems [18,32]. We show that formation of atomic scale wires in one tunnel gap can lead to an avalanche of subsequent wire formation, and consequent increases in conductance that mimic the increase in connectivity of biological neural systems in response to inputs. We then present a detailed discussion of the data including a comparison with biological and experimental solid-state systems, and conclude with a discussion of possible variations on the experimental system that would show neuromorphic behavior, including molecular systems.

Since the literature contains a number of definitions, for the sake of clarity in the reminder of this paper we wish to distinguish between STP and LTP of individual synapses [8] and potentiation of a pathway through a network (e.g., of neurons and synapses). We will use the word potentiation to refer to the latter, i.e., the sensitisation of a pathway, corresponding to a memory response in the system of interest (see Ch. 6 in Ref. [14]). The potentiation of the network results from an avalanche of neuronal connections [23].

\section{SIMULATION DETAILS}

We focus on simulations of overlapping disks for a system size of $200 \times 200$ particle diameters (chosen to provide the best 
(a)

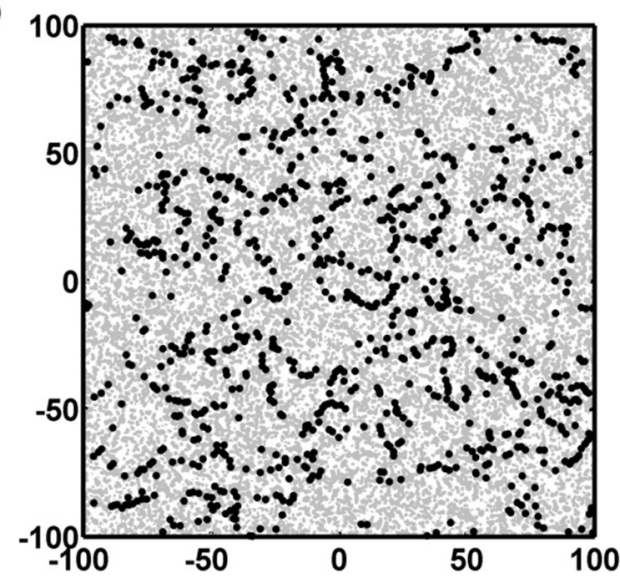

(b)

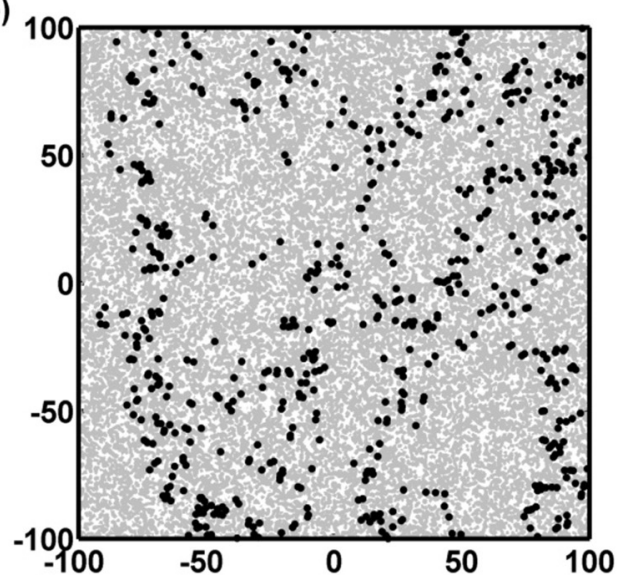

FIG. 1. Map of $200 \times 200$ system of overlapping disks (gray) with locations of switching events shown using black dots. The electrodes (not shown) are at the left and right edges of the system. $V_{\max }=1 \mathrm{~V}$ and $p=0.55$ (left), $p=0.65$ (right). At higher coverages the larger size of the connected groups [33] means that there are fewer tunnel or switching junctions between groups and a much higher conductance prior to the switching process (by up to 8 orders of magnitude); it also means that there are fewer locations at which switching can occur.

tradeoff between computational time and finite size effects). The disks are allowed to have random positions and we have previously shown that this continuum percolation model provides a good representation of the random assemblies of nanoparticles that are of interest here [33-35]. Reference [33] provides a more detailed comparison with (and discussion of) the experimental system-note in particular that when the particles touch they are strongly connected electrically, as are overlapping disks.

The key difference between the present simulations and standard models of continuum percolation [36] is that here we explicitly allow tunneling as in Ref. [33]. Tunneling processes [37,38] and related soft percolation problems [39] have been studied for some time, but it has only recently been demonstrated $[33,40,41]$ that when tunneling is allowed the system conductance depends exponentially on surface coverage $p$ below the percolation threshold, $p_{c}=0.676336$ [42] and that for $p>p_{c}$ the system conductance obeys the power law:

$$
G \propto\left(p-p_{c}\right)^{t},
$$

where in two dimensions $t$ takes the universal [43] value 1.3.

We focus here on the regime $p<p_{c}$, i.e., below the percolation threshold (see Fig. 1), where the conductance of the system is due to tunneling across gaps between the groups of connected particles. The connections between overlapping particles are assumed to have negligible resistance [33]. Each gap is assigned a conductance,

$$
G_{i}=A \exp \left(-\beta L_{i}\right),
$$

where A and $\beta$ are constants and $L_{i}$ is the size of the gap ( $A=1$ for convenience and $\beta=100$ ). The conductance of the network is then calculated by solving for the voltages at each node, with boundary conditions set by the voltage difference between the electrodes on either side of the system. The particles are assumed to be monodisperse [44] with diameter 1 and all distances (e.g., $\left.L_{i}, \beta\right)$ are measured in units of the particle diameter.
To study the voltage response of the network we ramp the voltage across the system in a triangular ramp from $V=0$ to $V_{\max }$ and back to $V=0$, with constant step size $0.025 \mathrm{~V}$. Hence the number $\left(N_{V}\right)$ of voltage steps is sufficient to characterize both the voltage at any time and the time since the start of the ramp, and we plot all data as a function of $N_{V}$. We simulate the switching process by first identifying the smallest gaps between each pair of groups. We then identify those gaps with electric fields larger than a chosen threshold (here $E_{\text {th }}=0.9$ ) [45] which are then replaced with a large conductor $\left(G_{\text {Ohmic }}=\right.$ $10 \Omega^{-1}$ ) with probability $P_{\uparrow}$. This process simulates the formation in the tunnel gap of an atomic wire, which occurs due to either electric-field-induced surface diffusion (EFISD) or electric- field-induced evaporation (EFIE) [27,46-48]. We then recalculate the conductance of the network $G$. At the next voltage step the process is repeated; the voltage ramps continue until the system conductance converges-typically to within $0.1 \%$ of the value on the previous step. The probability $P_{\uparrow}$ accounts for the stochastic nature of the switching process and we have performed simulations for $P_{\uparrow}$ in the range $0.1 \%-100 \%$. For all our simulations we record the cumulative number of replacements (i.e., switching events) $N_{R}$, as well as $G$ and the current flowing $I$. Note that we have chosen to focus on voltage ramps for consistency with the experimental protocol used in Ref. [33], but discuss dc applied voltages and voltage pulses in Sec. IV D.

We note that in this model the conductance can only increase: These simulations are a deliberate simplification of the experimental situation where decreases in conductance are also observed due to breaking of the atomic scale wires by electromigration [27]. It is intuitively clear that if both creation and breaking of connections is possible there will be random switching of the conductance around some mean value that is determined by the relative probabilities of the two types of events. By focusing on the simplified model we are able to more clearly demonstrate potentiation of the network, i.e., neuromorphic behavior of the system. The "noise" generated by breaking connections serves only to complicate matters, as we show in Sec. III C when $I$ exceeds 
a current threshold $I_{\text {th }}=0.1$ we allow breakage of previously establish connections with probability $P_{\downarrow}$.

We note that the present simulations are very different from those in Ref. [27]. There it was shown that the probability of obtaining a quantized system conductance when a quantized local conductance was introduced in a tunneling gap increased dramatically as $p$ approached $p_{c}$; in that case we replaced the tunnel gaps with the highest field with quantized conductors but did not iteratively solve for a new system conductance, nor did we change $\mathrm{V}$ at all. There was no possibility of a cascade of switching events and no attempt to observe neuromorphic behavior. Here the situation is different because we allow successive replacements of tunnel gaps with Ohmic conductors (and ignore any possible quantization effects) which allows potentiation.

Finally we note that the values of the system conductance in our simulations range from $G \sim 10^{-11} \Omega^{-1}$ to $10^{2} \Omega^{-1}$; these values are determined by our choice of $A, \beta$, and $G_{\text {Ohmic }}$. Our choice of $G_{\text {Ohmic }}=10 \Omega^{-1}$ is somewhat arbitrary but it is of course possible to scale our results to the quantized [28-30] value $G_{0}=e^{2} / h=1 / 25.9 \mathrm{k} \Omega$ that is obtained experimentally for atomic scale wires [27]. The value $\beta=400$ corresponds to our previous experiments [33] but in the simulations we choose $\beta=100$ as this allows calculation of tunneling behavior (i.e., increases the system conductance) over a larger range of $p$. This means that the ratio of the tunneling and wire conductances is smaller, and the neuromorphic behavior somewhat less dramatic, than could be expected in experiments.

\section{RESULTS}

Figure 2 shows the current through the percolating system of nanoparticles in response to the applied sawtooth voltage

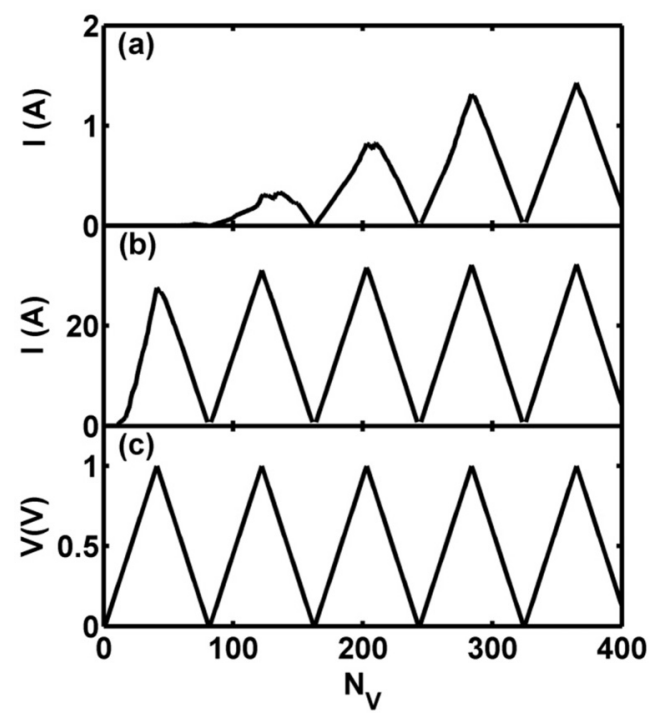

FIG. 2. Current flowing between the electrodes as a function of time (which corresponds to the number of voltage steps $N_{V}$ ) for $P_{\uparrow}=10 \%$ and (a) $p=0.55$, (b) $p=0.65$. (c) Corresponding voltage ramp with $V_{\max }=1 \mathrm{~V}$ and $V_{\text {step }}=0.025$. The increase in current as a function of applied voltage and in subsequent voltage cycles is due to the insertion of high conductances (representing atomic wires) in tunnel gaps between particles when the electric field in the gap exceeds a defined threshold $E_{\mathrm{th}}$.

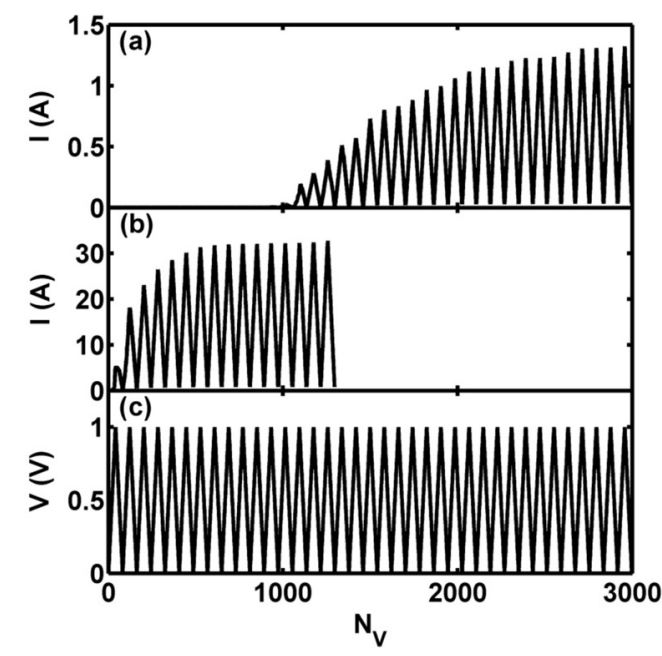

FIG. 3. As for Fig. 2 but with $P_{\uparrow}=1 \%$. (a) $p=0.55$, (b) $p=$ 0.65 , (c) corresponding voltage ramp. The data in (b), as in many other figures below, terminates when the conductance converges to within $1 \%$.

with $V_{\max }=1 \mathrm{~V}$ and $P_{\uparrow}=10 \%$. For low coverages $[p=0.55$, Fig. 2(a)] the initial conductance is low (invisible on this linear scale during the first cycle of the voltage ramp) because all current flow is via small tunneling conductors. It is immediately clear, however, that the current increases in response to the increasing applied voltage during the second voltage cycle, as is expected when the tunneling conductors are replaced by high conductances representing atomic scale wires. Subsequent cycles of the voltage ramp cause a further increase in the current until after the fifth cycle the network conductance is dominated by the high conductances $G_{\text {Ohmic }}$ and saturates. At higher coverages $[p=0.65$, Fig. 2(b)] significant current is observed immediately and the conductance saturates much more quickly. This is because the tunneling gaps are both fewer and smaller and so the applied voltage causes a more dramatic increase in electric field, resulting in more switching events even at low voltages during the first cycle of the voltage ramp.

Figure 3 shows similar results for $P_{\uparrow}=1 \%$. Here, because the switching probability is smaller, a larger number of voltage cycles are required but eventually the conductance again saturates. While Figs. 2 and 3 clearly show the increase in current with time it is evident that for much of the time the current flow tracks the sawtooth voltage waveform: The neuromorphic behavior is shown more clearly in plots of the system conductance $G$ versus $N_{V}$, as in all further plots below. In addition, we use logarithmic plots to make the small changes in conductance in earlier cycles of the voltage ramp [e.g., in Figs. 2(a) and 3(a)] visible. We emphasise that $N_{R}$ and $G$ provide complementary ways of characterizing the level of potentiation in the network.

\section{A. Finite $\boldsymbol{P}_{\uparrow}$}

As noted above $P_{\uparrow}$ allows us to account for the stochastic nature of the switching process, i.e., for the fact that atomic scale wires are not formed completely deterministically. On a microscopic scale $P_{\uparrow}$ accounts for different local environments around each tunnel gap and the time taken for electric field 

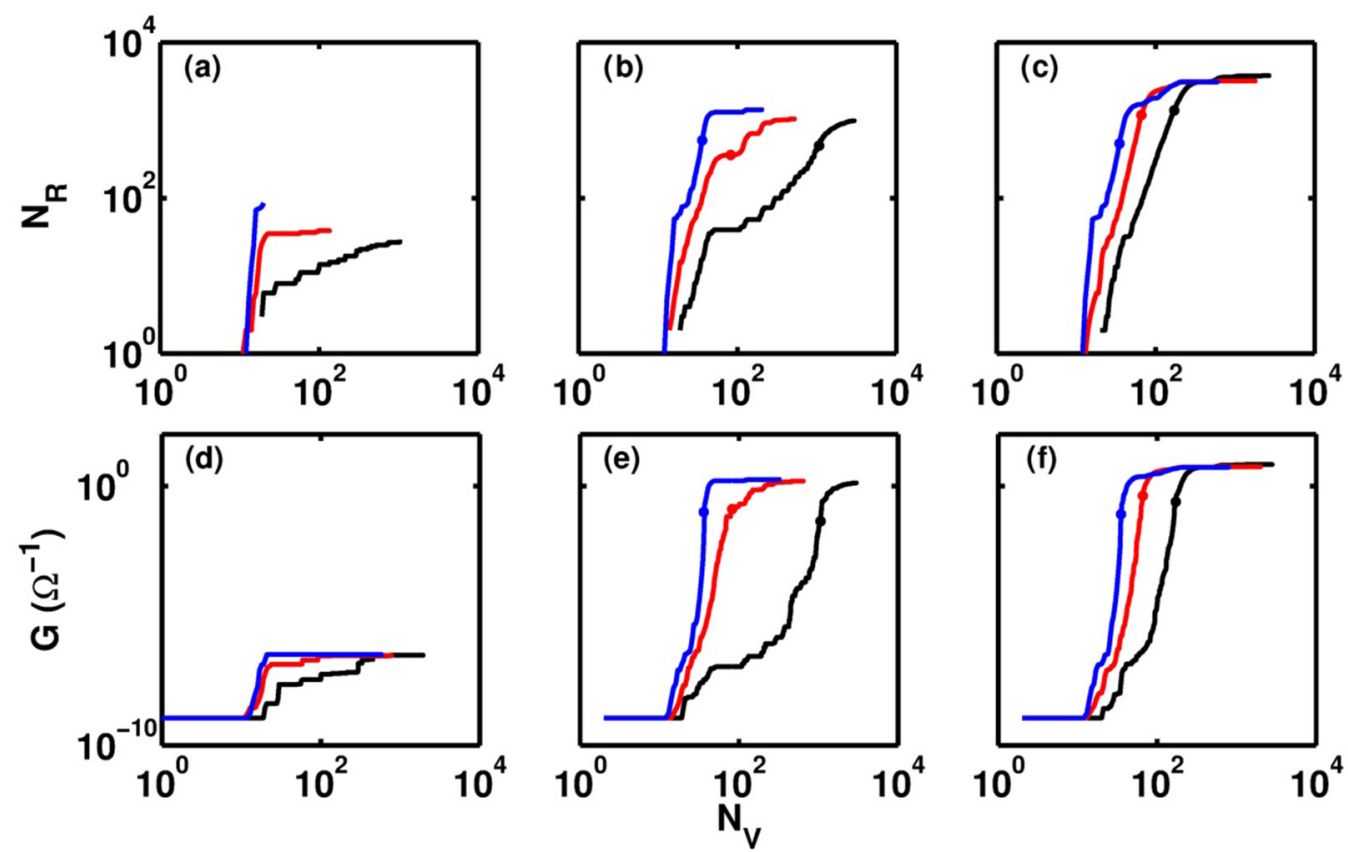

FIG. 4. (Color online) Number of tunnel junctions replaced $\left(N_{R}\right)$ and system conductance $(G)$ as a function of time (number of voltage steps $N_{V}$ ) for $p=0.55$ and $P_{\uparrow}=1,10$, and $80 \%$ (black, red, and blue curves, respectively, and from right to left). (a) and (d) $V_{\max }=0.5 \mathrm{~V}$, (b) and (e) $V_{\max }=1 \mathrm{~V}$, (c) and (f) $V_{\max }=5 \mathrm{~V}$. Colored dots mark the points at which the last tunneling conductor on the primary conduction path between the contacts is replaced by an Ohmic conductor $G_{\text {Ohmic }}$.

effects to result in formation of the atomic wire [46-48], a process which is not completely understood.

We begin by focusing on systems with $p=0.55$. Figure 4 shows the evolution of both $N_{R}$ (top row) and $G$ (bottom row) with time (i.e., $N_{V}$ ) for a range of values of $V_{\max }$ and of $P_{\uparrow}$. In each case, it is clear that an increase in $P_{\uparrow}$ leads to a faster increase in the number of replacements and consequently a faster increase in the conductance. The maximum conductance reached for each set of curves is clearly highest for the largest $V_{\max }$, since $E_{\text {th }}$ is exceeded in a larger number of tunnel gaps. However, the full effect of the maximum voltage is actually more subtle. At low $V_{\max }$ [Figs. 4(a) and 4(d)], $E_{\text {th }}$ is only ever exceeded for a relatively small number of tunnel gaps and so the number of replacements is modest and the conductance saturates at small values (still in the tunneling regime). At higher $V_{\max }$, once $E_{\mathrm{th}}$ is exceeded there is a nearly exponential initial increase in both $N_{R}$ and $G$ until eventually the last tunneling conductor on the primary conduction path between the contacts is replaced (the points marked by colored dots in Fig. 4; these points define a critical number of voltage steps $N_{V}^{C}$; see below). Note that at later times $N_{R}$ saturates both because most of the relevant tunnel gaps have already been replaced and because the existence of an Ohmic conductance path means that the voltage distribution across the system is relatively uniform and there are few opportunities to newly exceed $E_{\text {th }}$.

Note that the horizontal scale in Fig. 4 is a little deceptive and needs careful attention for two reasons: (i) It is logarithmic and so emphasizes changes at small $N_{V}$-Fig. 5 shows the data from Figs. 4(c) and 4(f) on a linear horizontal scale which emphasizes the exponential initial increase in $G$ and $N_{V}$; and (ii) because $V_{\text {step }}$ is constant, the length of each voltage cycle
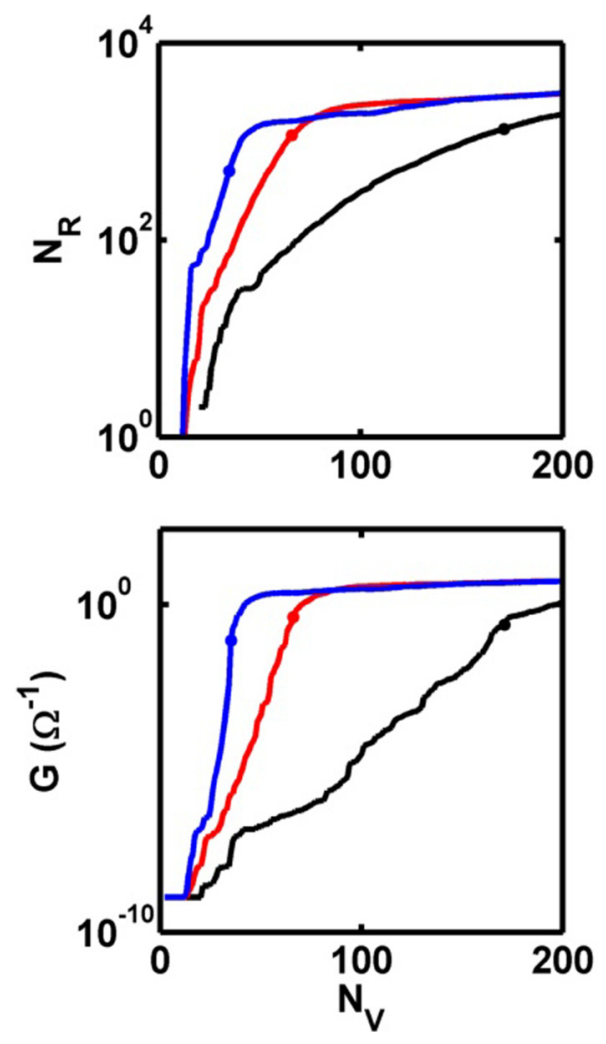

FIG. 5. (Color online) Data from Figs. 4(c) and 4(e) (i.e., $p=$ $\left.0.55, V_{\max }=5 \mathrm{~V}\right)$ replotted on a linear horizontal scale so as to allow the behavior at small $N_{V}$ to be seen more clearly and to emphasize the nearly exponential initial increase in $G$. Colored dots mark the points at which the last tunneling conductor on the primary conduction path between the contacts is replaced by an Ohmic conductor $G_{\text {Ohmic }}$. 

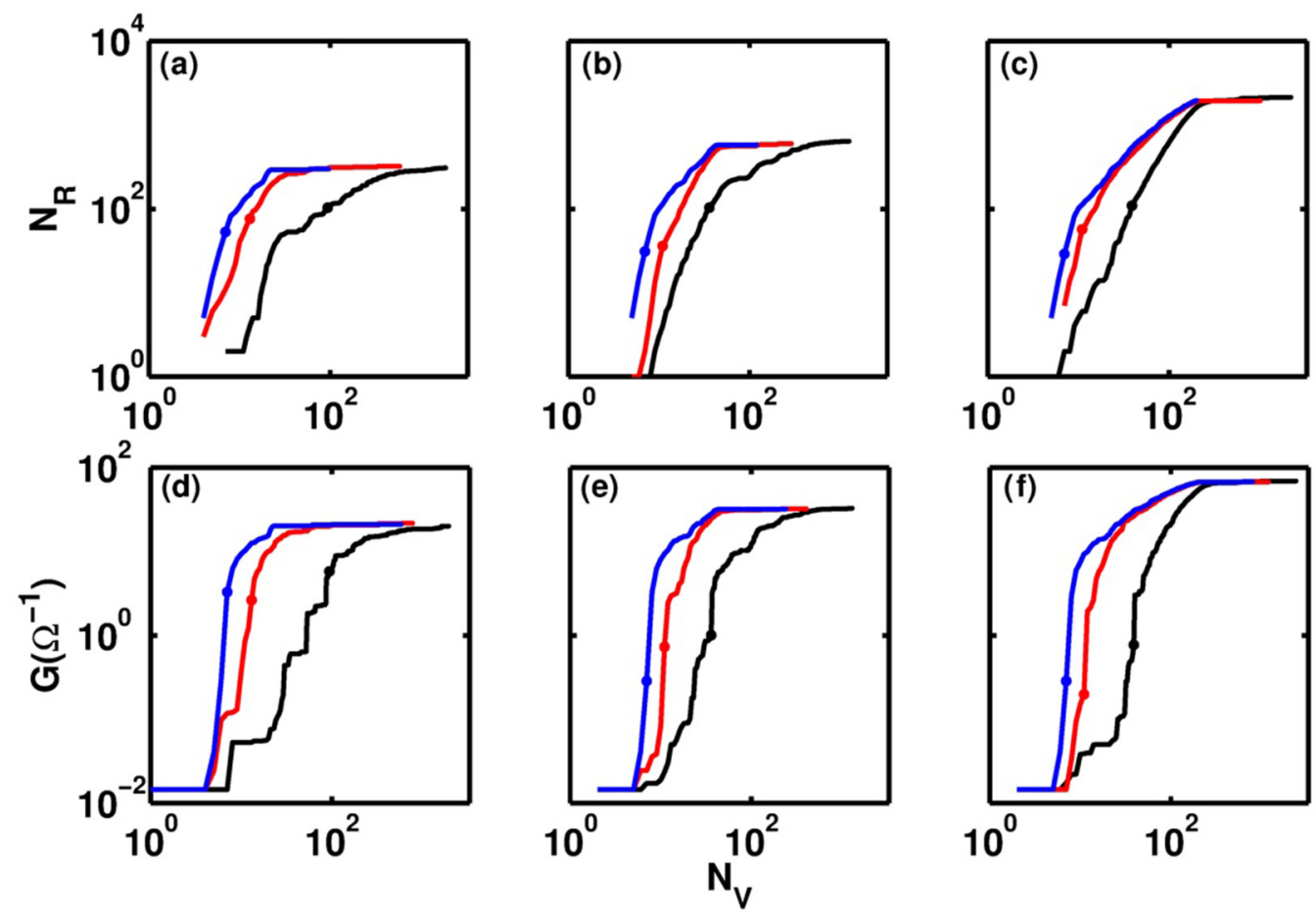

FIG. 6. (Color online) As for Fig. 4 but for $p=0.65$.

is proportional to $V_{\max }$ meaning that, for example, the data in Figs. 4(a) and 4(d) cover a number of cycles whereas the data in Figs. 4(c) and 4(f) cover only one voltage cycle. Figure 5 shows that the apparently S-shaped conductance curves in Fig. 4 in fact comprise a nearly exponential region followed by a region of saturation: $G$ saturates because $N_{R}$ is saturating and because of the functional form of Eq. (1) as discussed further in Sec. III B 1.

Figure $6(p=0.65)$ shows similar general trends to Fig. 4, although note the dramatic decrease in the range of conductivities (change in $G$ scale) compared to Fig. 4 . The key difference is that because the coverage is higher, the initial conductance $G_{\min }$ for each curve is many orders of magnitude larger than in Fig. 4 (while the final conductance $G_{\max }$ is only slightly higher). This is because $G_{\min }$ is associated with the tunneling regime where the conductance changes exponentially with coverage [33] whereas $G_{\max }$ is associated with the regime governed by Eq. (1).

These differences are made clearer in Fig. 7 where we directly compare the conductance as a function of number of voltage steps for $p=0.55$ and $0.65, V_{\max }=1 \mathrm{~V}$ and $5 \mathrm{~V}$, and $P_{\uparrow}=1 \%$ and $40 \%$. Clearly $G_{\min }$ and $G_{\max }$ are smaller for $p=0.55$ than for $p=0.65$ but another important difference is that the number of replacements required to create an Ohmic path is larger for $p=0.55$. This larger critical value of $N_{V}^{C}$ is analogous to a new percolation threshold [for the percolating system comprising tunnel gaps (empty sites) that are being replaced by Ohmic conductors (filled sites)]—see Sec. III B 1.

$$
\text { B. } P_{\uparrow}=100 \%
$$

Clearly the conductance in Figs. 4, 5, 6, and 7 is stochastic: There is a probabilistic element to the simulations $\left(P_{\uparrow}\right)$ which means that different tunnel gaps are replaced by Ohmic con- ductors on each run and that the precise shapes of each curve are different from run to run. (In addition we must be clear that any given realization of a system with a certain $p$ will yield slightly different data-the precise sizes and positions of the steps in $G$ versus $N_{V}$ plots will be different. Nevertheless the trends for each realization are the same and the $G_{\min }$ and $G_{\max }$ values are very consistent for the large system sizes studied here.) This stochastic element can be removed by setting $P_{\uparrow}=100 \%$ as in Fig. 8(a), which allows a simpler comparison of the effect of $p$ on the overall size of the neuromorphic effect: The ratio $G_{\max } / G_{\min }$ clearly decreases with increasing $p$ and at large $p$ a smaller number of voltage steps are required to achieve neuromorphic behavior. Perhaps most importantly, at low $p$ the number of voltage steps required to complete

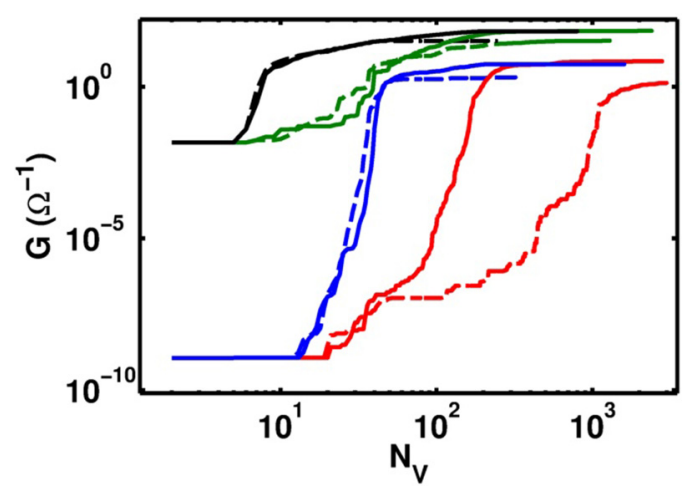

FIG. 7. (Color online) Direct comparison of conductance as a function of time $\left(N_{V}\right)$ for a range of parameter values: $p=0.55$ and $0.65, V_{\max }=1 \mathrm{~V}$ (dashed lines) and $5 \mathrm{~V}$ (solid lines), and $P_{\uparrow}=1 \%$ and $40 \%$. (From right to left) $p=0.55, P_{\uparrow}=1 \%$ (red); $p=0.55$, $P_{\uparrow}=40 \%$ (blue) $p=0.65, P_{\uparrow}=1 \%$ (green); $p=0.65, P_{\uparrow}=40 \%$ (black). 

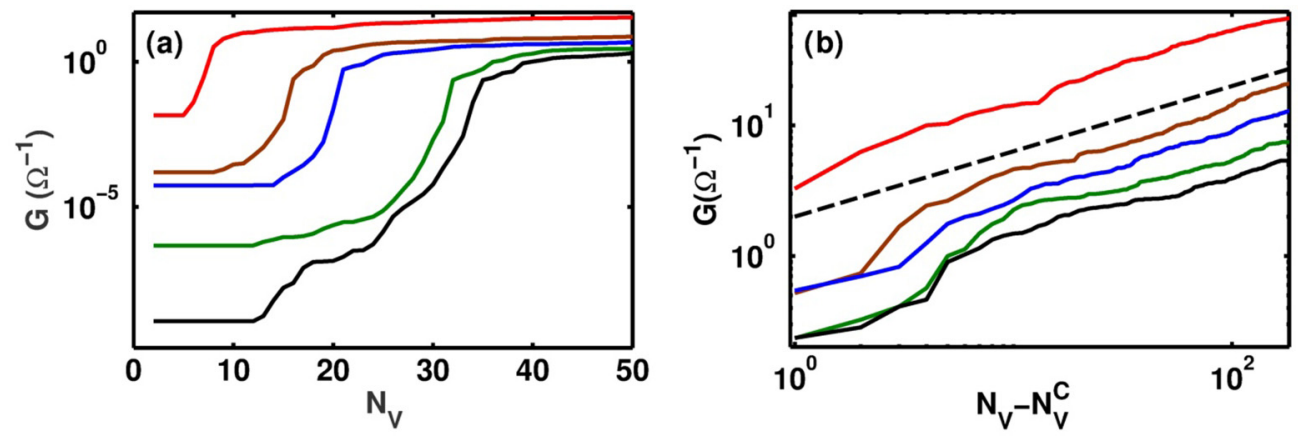

FIG. 8. (Color online) (a) $G$ as a function of $N_{V}$ for $P_{\uparrow}=100 \%, V_{\max }=5 \mathrm{~V}$. (From bottom to top) $p=0.55,0.57,0.60,0.62,0.65$. (b) The same data plotted as function of $N_{V}-N_{V}^{C}$ in order to show the power law variation of the conductance discussed in the text. The dashed line indicates a slope of 0.5 .

the process is much greater (i.e., the width of the transition is larger) and this means that the number of switching events that occur during potentiation is greater-see discussion below.

The effects of changing the probability of formation of a connection and maximum applied voltage are as intuitively expected, i.e., increases in $P_{\uparrow}$ and $V_{\max }$ both increase the rate at which connections are formed, and consequently the rate of increase of conductance is also increased. However, the response to the ramped voltage does not scale trivially with time (equivalently $N_{V}$ ): In periods of decreasing voltage the rate of formation of connections is limited. Hence it is not possible to simply scale the data in Figs. 4 and 6 onto Fig. 8(a) even though the response to the voltage ramps is essentially the same.

\section{Power law regime}

Figure $8(\mathrm{~b})$ shows the data for $P_{\uparrow}=100 \%$ on an expanded scale, revealing a power law behavior in the conductance. This power law regime is visible at all coverages and particularly at high probabilities but is obscured in Figs. 4 and 6 by the choice of vertical scale. This behavior is observed for $N_{V}>N_{V}^{C}$ and is clearly distinct from the exponential behavior in Fig. 5 for $N_{V}<N_{V}^{C}$.

Above the percolation threshold $N_{V}^{C}$, where the switched junctions and their associated groups span the system, this power law region can be explained qualitatively by the existence of an Ohmic spanning group which grows in a fashion which is dictated by the underlying structure of the connected switchable elements (which are themselves given by the underlying percolating structure of the film). After the first Ohmic connection across the film is formed at $N_{V}^{C}$, further cycling of the voltage links a subset of the remaining groups to the spanning group, gradually approaching a saturation point, at which the conductance plateaus. The random insertion of $G_{\text {Ohmic }}$ resistors into the network of tunneling gaps is a kind of additional percolation process, i.e., percolation of connections on top of the percolating network of particles.

The slope of the data in Fig. 8(b) is approximately 1/2, as shown by the dashed line. It is not clear why the slope takes this value [the power law exponent in Eq. (1) is $t \sim 1.3$ ] but it is also not clear how to relate $N_{V}$ to $p$ in Eq. (1). Further investigation is required, including a systematic study of the system size dependence of this exponent, but we speculate that it should be possible to define an effective coverage $p^{*}$ that is a function of both $p$ and $N_{V}$ and that some progress might be made by equating $\left(p^{*}-p_{c}^{*}\right)^{t}$ to $\left(N_{V}-N_{V}^{C}\right)^{1 / 2}$. However, Sec. IV B shows that $N_{V}^{C}$ is coverage dependent and this problem requires further detailed analysis.

\section{More realistic simulations including wire breaking $\left(\boldsymbol{P}_{\downarrow}\right)$}

The simulations discussed to this point include only replacements of tunneling conductors with the Ohmic conductors $G_{\text {Ohmic }}$ (representing the formation of atomic wires) so that the neuromorphic behavior is clearly manifested as an increase in conductance. Now we demonstrate the effect of including in the model the possibility that the atomic wires formed can subsequently be broken by electromigration [27]. We assume that when the current in an atomic wire exceeds a threshold value $I_{\text {th }}=0.1$ the wire has a well-defined probability of being broken $P_{\downarrow}$ at each voltage step. When a wire is broken it is assumed that the original gap between particles reappears (experimentally it is clear that this need not be true, but this is a reasonable first approximation).

We begin by considering an example with $P_{\uparrow}=1 \%$ and the extreme case of $P_{\downarrow}=100 \%$. Figures 9(a) and 9(b) show the

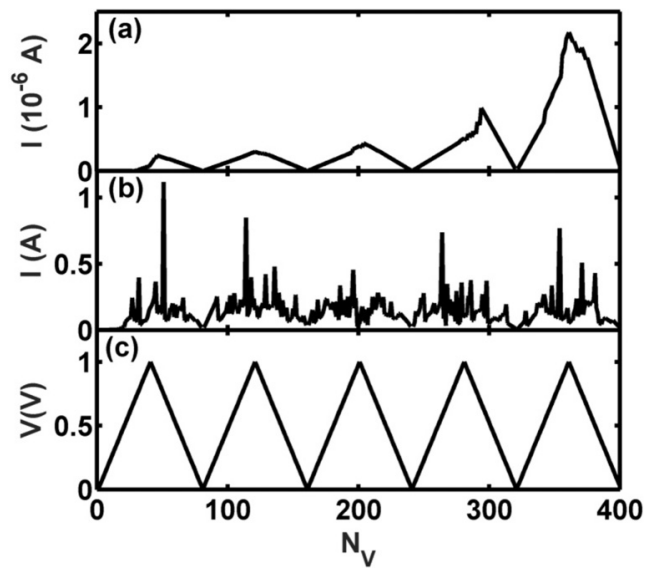

FIG. 9. $G$ versus $N_{V}$ when both creation (with probability $P_{\uparrow}=$ $1 \%$ ) and breaking (with probability $P_{\downarrow}=100 \%$ ) of atomic wires is allowed in the simulation (at $E_{\text {th }}=0.9$ and $I_{\text {th }}=0.1$, respectively). (a) $p=0.55$, (b) $p=0.65$, (c) corresponding voltage ramp. Note the change in scales from (a) to (b). 
response of systems with $p=0.55$ and 0.65 , respectively, to a voltage ramp $\left[V_{\max }=1 \mathrm{~V}\right.$, Fig. 9(c)]. Interestingly, despite the fact that $P_{\downarrow}=100 \%$, Fig. 9(a) still shows clear neuromorphic behavior in the form of increases in current from cycle to cycle. This is because of the large number of tunneling gaps that exist in series in low coverage samples, which means that the current flowing through the atomic scale wires that are formed is limited by the series resistance and the threshold $I_{\text {th }}$ is not reached. Hence the data in Fig. 9(a) is actually identical to what would be seen on an expanded scale in the first few cycles of Fig. 3(a) (note the difference in vertical scales). In contrast the formation of atomic wires during the first cycle in Fig. 9(b) results in much higher currents [because the number of series tunnel gaps is smaller-compare Figs. 1(a) and 1(b)] and electromigration immediately starts to remove the Ohmic conductors for which $I>I_{\text {th }}$. Hence, even in the second voltage cycle, a dynamical equilibrium is obtained as wires repeatedly form and break, and the average current flowing in the subsequent cycles is approximately constant.

The more realistic case where $P_{\downarrow}=1 \%$ is shown in Fig. 10 . As in Fig. 9(a), for $p=0.55$ Fig. 10(a) shows an increase in the tunneling current from cycle to cycle but the large series resistance means that electromigration is irrelevant. As we move to higher coverages [Figs. 10(b) and 10(c)] the increases in current from cycle to cycle are faster, and the maximum current and conductance increase with $p$. Initially electromigration has only a limited impact and so it takes some time for a dynamic equilibrium to be established (after a relatively large number of cycles). The behavior in Figs. 10 (b) and 10(c) is quite comparable to the behavior reported in other experimental realizations of neuromorphic systems [6]. In particular, in this regime the behavior is very similar to that associated with Aviziensis et al. with recurrent connections and feed forward mechanisms (see, for example, Fig. 4 in Ref. [6]). Finally, we note that when the devices are operated in the tunneling regime [as in Figs. 9(a) and 10(a)], the inherent

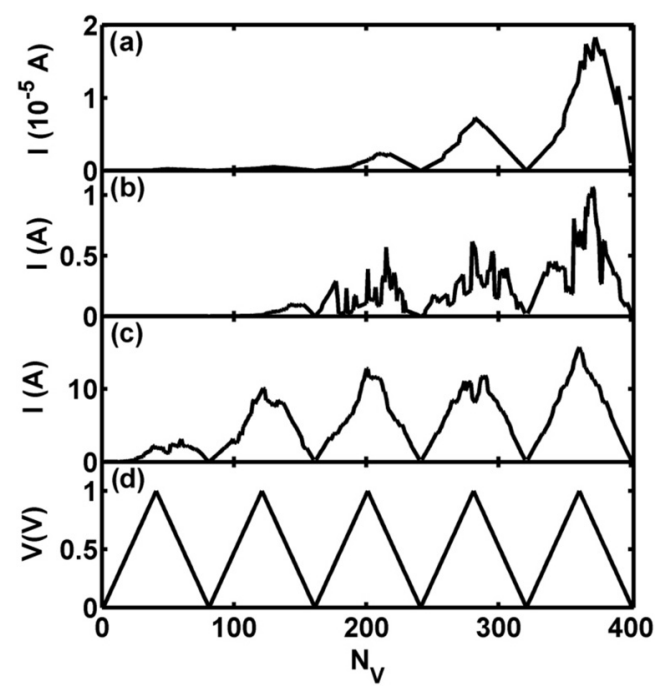

FIG. 10. Same as for Fig. 9 except with $P_{\downarrow}=1 \%$. (a) $p=0.55$, (b) $p=0.60$, (c) $p=0.65$, (d) corresponding voltage ramp. Note that (a) is on a different vertical scale from (b) and (c). nonlinearity may be useful in providing functionality similar to that required for reservoir computing [7,24,25].

\section{DISCUSSION}

When we include both formation and breaking of atomic scale wires our model produces realistic potentiation behavior (Fig. 10), demonstrating that percolating films of nanoparticles have potential applications in neuromorphic devices. Implementation of neuromorphic devices will require multicontact geometries $[6,8]$ where inputs to particular contacts potentiate different pathways within the system, and there are a large number of possibilities for variations on the scenario considered here. In particular we envisage the use of switching molecules (through chemical functionalization of the nanoparticles) and other memristive elements. The extension of the present model to such geometries and differing switching elements should be straightforward; we discuss these variations and other relevant effects in turn below.

\section{A. Geometrical effects}

We find potentiation (in the sense discussed in the introduction) of the nanoparticle networks for all surface coverages studied; we have presented data for $p=0.55$ and 0.65 but similar behavior is observed for other coverages in this range. The size of the neuromorphic effect (change in $G$ ) is significantly more dramatic for systems with low particle coverages because the networks are more complex. As $p \rightarrow p_{c}$ the size of the connected groups of particles increases and approaches the size of the simulated system which means that the number of tunneling connections that must be traversed in order to span the system becomes smaller: This means that there are fewer locations at which atomic scale wires could be formed, and hence that potentiation involves fewer switching effects [the total number of tunneling conductors $N_{R}$ that are replaced at the end of the process is about an order of magnitude higher in Fig. 4(c) compared to Fig. 6(c)]. The average size of the gaps decreases with increasing $p$ [33] and together with the smaller number of gaps this means the average electric field in each gap is higher, which means that electric field driven switching [27,46-48] is more likely for higher $p$. In contrast, we expect that for coverages lower than those studied here the network will be simplified and become similar to a random system of dopants [33,49]; this regime is not experimentally accessible in the nanoparticle films of interest because the current flowing falls below the noise threshold.

\section{B. Critical point for secondary percolation}

As discussed above the critical number of voltage steps $\left(N_{V}^{C}\right)$ required to achieve an Ohmic connection across the system is highlighted by colored dots for each of the curves in Figs. 4 and 6. Figure 11 shows the dependence of $N_{V}^{C}$ on coverage $p$ for several different values of $P_{\uparrow}=1 \%$ and $V_{\max }$. The further the system is from the percolation threshold, the more voltage steps (and more replacements) are needed to achieve an Ohmic connection. Interestingly the three data sets for which the chosen parameters avoid significant periods where no switching occurs during decreases of the voltage 


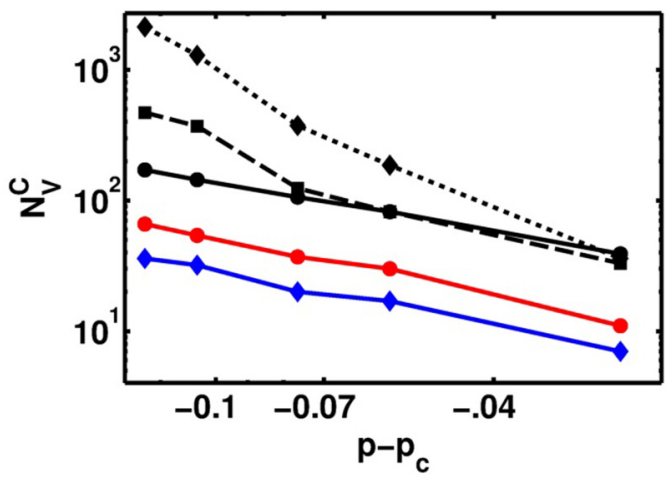

FIG. 11. (Color online) Dependence of critical number of steps required to create an Ohmic connection across the system $N_{V}^{C}$, on coverage $p$, extracted from the data in Figs. 4 and 6. (Top to bottom) $P_{\uparrow}=1 \%$, at $V_{\text {max }}=1 \mathrm{~V} ; P_{\uparrow}=1 \%$, at $V_{\text {max }}=2 \mathrm{~V} ; P_{\uparrow}=1 \%, V_{\text {max }}=$ $5 \mathrm{~V} ; P_{\uparrow}=10 \%$, at $V_{\text {max }}=5 \mathrm{~V} ; P_{\uparrow}=80 \%$, at $V_{\max }=1 \mathrm{~V}$. Lines serve only to connect the data points.

ramps (plotted with solid lines) are each close to a power law with exponent 1 , i.e., $N_{V}^{C}$ varies approximately linearly with $p-p_{c}$. The higher the probability of creating an atomic wire, the smaller is $N_{V}^{C}$ : we find that $N_{V}^{C}$ scales according to the value of $P_{\uparrow}$. The effect of $V_{\max }$ on $N_{V}^{C}$ is more subtle-comparison of the three data sets for $P_{\uparrow}=1 \%$ but different voltages in Fig. 11 makes it clear that there is no simple scaling with voltage; this effect requires further exploration.

\section{Comparison with biological neural networks}

There are many aspects of the present solid-state system that are different to real-world biological systems. Neurons exist in a complex environment comprising astrocytes, glial, and other cells, and synapses rely on biochemical mechanisms that have no simple solid-state analog. These are important neurologically - for example, the biochemical modification of neural function by drugs - but our goal is not to replicate such functionality in solid-state systems. Rather, here the focus is on a demonstration of potentiation as a necessary (but not sufficient) condition for neuromorphic computing. Further simulations, for example, those which include more complex switching elements, multiple synapses between neurons, multiple electrodes, more sophisticated pulsed inputs, and multiple inputs with opposite polarity [50] are necessary to explore the latter.

One might have expected that the large fractal groups that are present for $p$ near $p_{c}$ would maximize the opportunity for interconnectivity between groups. This may be a key difference between the present system and real neural networks: The present system is inherently two-dimensional and this dimensionality limits the neuromorphic potential of the system. We suggest that an equivalent three-dimensional system would allow greater opportunities for connections between non-neighboring groups of particles. A significant outstanding challenge is to be able to fabricate quasi-threedimensional systems that exhibit large fractal structures: Such systems should exhibit even more interesting neuromorphic behavior.
A further difference between the present system and biological neural systems is that there it is possible to form more than one connection between neural cells, i.e., multiple synapses between the same pair of neurons resulting in an enhanced response to the stimulus from the incoming axon. Here, because of our choice of the value of $G_{\text {Ohmic }}$ and parameters in Eq. (2), if we create one connection between groups it shorts out any possible other tunnel gaps and (for the most part) removes the possibility of other replacements that would cause multiple connections between groups. The experimental system does, however, allow conductances with multiple levels; see Sec. IV E 1.

\section{Comparison with experiments \\ 1. Pulsed inputs}

In experiments on biological neurons and in neuromorphic computing one would expect to apply a series of voltage pulses (rather than the voltage ramps considered here) in order to cause potentiation. Numerically (i.e., in our simulations) providing a series of voltage pulses is identical to recording the response of the system to a constant (i.e., dc) voltage except that one should then interpret $N_{V}$ as the number of voltage pulses (with constant height) rather than the number of voltage steps. Figure 12 shows this for several different coverages and a constant voltage $V=1 \mathrm{~V}$. The results are qualitatively very similar to results from voltage ramps (compare with the dashed lines, corresponding to data from Figs. 4 and 6), but it is clear that the effect of the down ramps is to extend the amount of time required to achieve a given system conductance-switching events are relatively rare during down ramps.

\section{Experimentally controllable parameters}

Obviously the nanoparticle and system sizes, and applied voltages are all experimentally controllable parameters that will affect the switching behavior that will be observed. Hence, an additional point that should be made here is that because the switching depends on the local electric field in individual nanogaps, there is an opportunity in the experimental systems to tune the switching by using the nanoparticle size or the system size to tune the electric field that is actually present for any given applied voltage. In addition, EFIE and EFISD occur at different electric fields for different materials.

Put another way, the practical voltage range in which switching is observed will depend on nanoparticle size and

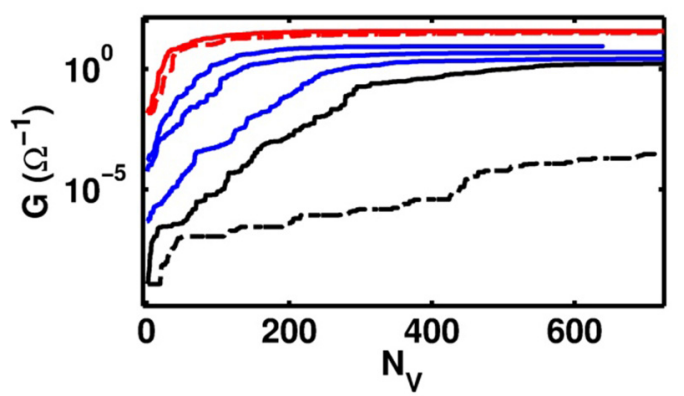

FIG. 12. (Color online) Conductance change at constant voltage ( $V=1 \mathrm{~V}$ ) for $p=0.55$ (black, right), 0.57, 0.60, 0.62, and 0.65 (red, left). Dashed lines are from ramped voltages for comparison. 


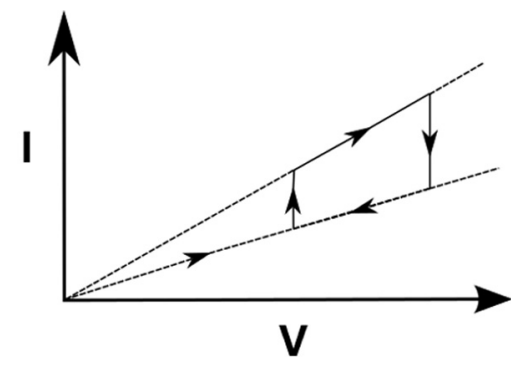

FIG. 13. Schematic I(V) curve for a single tunnel junction showing jump from an initial high resistance (tunneling) state to a low resistance state on formation of an atomic scale wire, and a hysteresis loop (solid lines) that results from a quasideterministic memristive behavior; see Sec. IVE. Note that while tunneling is a voltage-dependent process, for simplicity in this schematic we have assumed that even in the high resistance (tunneling) state the resistor still obeys Ohm's law at low V; different I(V) characteristics in the two states would change the shape of the curve but not the existence of a hysteresis loop. Any bias dependence will be unimportant in most applications because $G_{\text {high }}$ exceeds $G_{\text {low }}$ by many orders of magnitude - it is only the ability of the device to switch that is important to the observation of neuromophic behavior reported here.

the system size, as well as the choice of material. This is an important point, because it provides an opportunity in the nanoparticle system to controllably tune the level of potentiation that would be difficult to achieve in other systems.

\section{E. Memristive elements}

The tunnel junctions that are formed at gaps in the percolating film, and that switch to a highly conducting state on formation of an atomic scale wire, behave differently than memristors reported elsewhere [1,3]. These tunnel gaps are memristive in that the device history determines its state. Switching occurs at a well-defined threshold value (of the electric field [46-48]) but unlike the the memristive elements in Refs. [1,3] the junction cannot be switched back to the low conduction state by simply reversing the polarity of the bias voltage. Nevertheless this kind of "once only" switching provides sufficient functionality to enable potentiation of the network and a cascade of switching events, as described above.

As shown schematically in Fig. 13, once in the high conductance state, switching to the low resistance state occurs (due to electromigration) if the current exceeds some threshold value for long enough. We have modeled this above as a stochastic process that occurs with probability $P_{\downarrow}$, but it is clear that a slow voltage ramp that continues after the formation of the atomic wire will eventually cause the device to switch back to $G_{\text {low }}$ (if $P_{\downarrow}=1 \%$ then after 100 voltage steps, on average the device will be in the $G_{\text {low }}$ state). Hence, viewed over a long enough time frame, switching to $G_{\text {low }}$ occurs quasideterministically. Similarly, if the voltage is then ramped down at a similar rate (lower left-pointing arrow in Fig. 13), with the voltage remaining above $V_{\text {th }}$, a quasideterministic return to the high conductance state will occur due to formation of a new atomic scale wire. Hence if we define a protocol which requires that after this switching event the voltage is now ramped up again, we generate a clockwise hysteresis loop, as shown schematically in Fig. 13. Usually memristive hysteresis loops [1,3] are characterized by anticlockwise paths on an I(V) plot. The direction of the hysteresis loop changes some of the detail of the required protocols.

For completeness we note that (i) in the previous paragraph we have assumed that $I_{\text {th }}$ corresponds to a voltage greater than $V_{\text {th }}$, which appears to be the case in experiments [27]. In principle hysteresis loops could be either clockwise or counterclockwise depending on the relative positions of these thresholds. (ii) It should be possible to generate classic memristive hyseresis loops [1,3,8] using a further different protocol, i.e., after the initial increase in conductance shown in Fig. 13, if the voltage is decreased the current will be reduced so that electromigration can be eliminated. If we then choose to apply negative voltages sufficient current will be generated so as to break the connection and cause a return to the high resistance state, thus achieving memristive loops similar to those observed in other memristive devices $[1,3,8]$. In this protocol one uses large voltage pulses to switch states and small (subthreshold) voltage pulses to read the state of the devices.

\section{Wire width dependence-memristors with multiple conductance levels}

For simplicity, we have not considered here the possibility that the width of an atomic wire might change with time or in response to repeated inputs. Such changes are described in both Refs. [3,27] and could correspond to STP and LTP of individual synapses [8] (as emphasized in the introduction). Such variations of conductance will contribute an additional level of potentiation to the network: While adding further complexity to the simulations we believe that is likely to lead to qualitatively similar results to those already described above.

\section{Other memristive elements}

There are several straightforward adaptations of the system, relying on other memristive elements, that would allow alternate embodiments of neuromorphic devices. For example, we believe that devices fabricated with percolating silver nanoparticles could readily be sulphidized, which would open up the possibility of building neuromorphic devices based on percolating circuits using the electrochemical reduction approach of Refs. $[3,6]$. An additional alternative would be to incorporate switching molecules (for example, azobenzenes [51-53], rotaxanes [54,55], and other molecular switches [55-58]). Such hybrid percolating and molecular systems may allow additional and novel design parameters to be developed, and may well introduce more complex and interesting functionality.

\section{F. Avalanches and emergent complex dynamics}

The percolating system of interest is poised near a phase transition and so one of the long-term objectives of this work is to explore whether the emergent complex neural dynamics that are believed to occur in the brain can be reproduced in this solid-state system [23]. Figure 14 presents a time sequence that illustrates the spread of switching sites across the system in response to a constant voltage input. Clearly the potentiation 
(a)

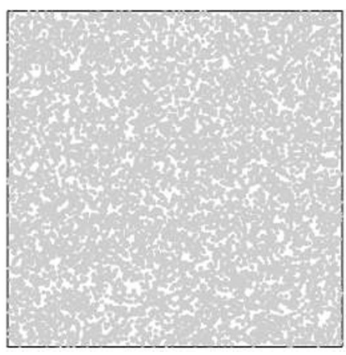

(b)

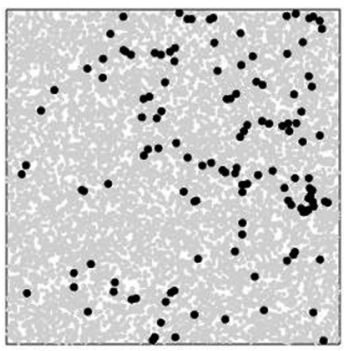

(c)

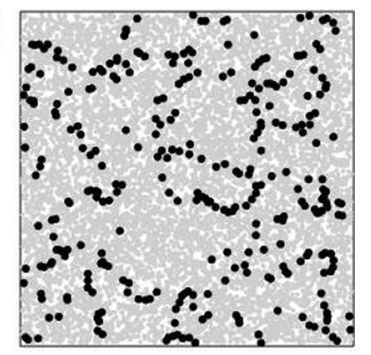

FIG. 14. Illustration of an avalanche of successive switching events resulting from application of a constant voltage (1 V). Locations of switching events (black dots) are shown for a $100 \times 100$ subset of a $200 \times 200$ system of overlapping disks (gray) with $p=0.55$ for $N_{V}=1$ (a), $N_{V}=2000$ (b), and $N_{V}=5500$ (c), corresponding to the conductivity data in Fig. 4(b).

of the network discussed above arises from a kind of avalanche of switching events; after formation of an atomic wire between one pair of connected groups of particles the applied potential is divided across other tunnel gaps in the network, increasing the local electric field and therefore increasing the likelihood of further switching events.

Our percolating networks exhibit many more small avalanches than large ones. Hence the present avalanche behavior has clear similarities to neuronal avalanches in biological systems, but further work is required to understand whether the avalanches in the present systems behave according to the five fundamental properties of neuronal avalanches that are identified in Ref. [23] as being consistent with criticality. The expected power law distribution in the size of cortical avalanches and lifetime statistcs $[59,60]$ are not straightforward to simulate in the present simulations, which do not include this dynamical behavior; in addition, further work is required to identify a measure of avalanche size that corresponds to that available from biological systems $[59,60]$. We speculate that the power law behavior discussed in Sec. III B 1 can be related to experimentally observed power laws for neuronal avalanche size and lifetime [59,60].

Despite the similarities it is clear that some aspects of the present system are not perfect analogs of the biological systems and that, for example, we cannot reproduce the biochemical complexity of the brain (see, e.g., Sec. IV C). Meanwhile other aspects of the present work-such as the limitation to only a single pair of electrical contacts to the sides of the percolating system - can obviously be extended in a relatively trivial way; indeed our next step is to develop simulations with multiple contacts to different local points on the edge of the network, as well as to contacts within the network, in conjunction with similar experiments that follow on from the work of Ref. [27].

Finally, it is worth mentioning that the present problem has some interesting elements in common with percolation models of electrical discharge $[61,62]$, in which conducting paths grow (due to ionization) through an insulating medium (air). The key difference between that work and the present results is that in Refs. [61,62] the probability of ionization is always determined by the local electric field at a site and so the occupation of sites in the lattice is not random. In the present continuum model the particles are deposited randomly resulting in a distribution of gap sizes [33] that is a representative function of coverage $p$. Hence $p$ governs the formation of the conducting paths and the potentiation behavior.

\section{CONCLUSIONS}

The present simulations focus on mapping potentiation in a percolating network and are a first step to a consideration of the questions of dynamics and criticality of the system [23]. We have shown that percolating films of nanoparticles exhibit neuromorphic behavior in the sense that application of a series of inputs causes potentiation of a pathway through the network, and a consequent direct transmission of subsequent signals to an output terminal. We have discussed in detail the response of the system to ramped, pulsed, and dc voltage inputs, which results from the formation of atomic scale wires in tunnel gaps in the network, and the influence of the probability of wire formation, as well as the effect of wire breaking due to electromigration effects. Finally, we have discussed possible future extensions of the model that might lead to a more direct comparison with biological systems; this future work will explore whether critical dynamics do indeed emerge in the present system with atomic scale switches, or other memristors, or indeed in other systems comprising memristors.

\section{ACKNOWLEDGMENTS}

The authors gratefully acknowledge financial support from the MacDiarmid Institute for Advanced Materials and Nanotechnology and the Marsden Fund, New Zealand.
[1] D. B. Strukov, G. S. Snider, D. R. Stewart, and R. S. Williams, Nature (London) 453, 80 (2008).

[2] L. Chua, IEEE Trans. Circuit Theory 18, 507 (1971).

[3] K. Terabe, T. Hasegawa, T. Nakayama, and M. Aono, Nature (London) 433, 47 (2005).

[4] M. Kaiser, Proc. R. Soc. London A 365, 3033 (2007).
[5] C. Mead, Proc. IEEE 78, 1629 (1990).

[6] H. O. Avizienis, C. Martin-Olmos, H. H. Shieh, M. Aono, A. Z. Stieg, J. K. Gimzewski, and A. V. Sillin, PloS ONE 7, e42772 (2012).

[7] A. Z. Stieg, A. V. Avizienis, H. O. Sillin, C. Martin-Olmos, M. Aono, and J. K. Gimzewski, Adv. Mater. 24, 286 (2012). 
[8] T. Ohno, T. Hasegawa, T. Tsuruoka, K. Terabe, J. K. Gimzewski, and M. Aono, Nat. Mater. 10, 591 (2011).

[9] T. Ohno, T. Hasegawa, A. Nayak, T. Tsuruoka, J. K. Gimzewski, and M. Aono, Appl. Phys. Lett. 99, 203108 (2011).

[10] S. H. Jo, T. Chang, I. Ebong, B. B. Bhadviya, P. Mazumder, and W. Lu, Nano Lett. 10, 1297 (2010).

[11] T. Chang, S. H. Jo, and W. Lu, ACS Nano 5, 7669 (2011).

[12] H. Kim, M. P. Sah, C. Yang, T. Roska, and L. O. Chua, Proc. IEEE 100, 2061 (2012).

[13] B. Linares-Barranco and T. Serrano-Gotarredona, Nature Precedings 2 (2009), http://precedings.nature.com/documents/ 3010/version/1.

[14] J. LeDoux, The Synaptic Self(Penguin Books, New York, 2002).

[15] R. A. Nawrocki, R. M. Voyles, and S. E. Shaheen, IEEE Trans. Electron Devices 61, 3513 (2014).

[16] L. Q. Zhu, C. J. Wan, L. Q. Guo, Y. Shi, and Q. Wan, Nat. Commun. 5, 3158 (2014).

[17] S. Peotta and M. Di Ventra, Phys. Rev. Appl. 2, 034011 (2014).

[18] H. O. Sillin, R. Aguilera, H.-H. Shieh, A. V. Avizienis, M. Aono, A. Z. Stieg, and J. K. Gimzewski, Nanotechnology 24, 384004 (2013).

[19] J. Borghetti, Z. Li, J. Straznicky, X. Li, D. A. A. Ohlberg, W. Wu, D. R. Stewart, and R. S. Williams, Proc. Natl. Acad. Sci. USA 106, 1699 (2009).

[20] A. Z. Stieg, A. V. Avizienis, H. O. Sillin, C. Martin-Olmos, M.-L. Lam, M. Aono, and J. K. Gimzewski, Jpn. J. Appl. Phys. 53, 01AA02 (2014).

[21] D. Monroe, Communications of the ACM 57, 13 (2014).

[22] M. Di Ventra and Y. Pershin, Electron. Lett. 50, 141 (2014).

[23] D. R. Chialvo, Nat. Phys. 6, 744 (2010).

[24] F. Rosenblatt, Psychol. Rev. 65, 386 (1958).

[25] W. Maass, T. Natschlager, and H. Markram, Neural Comput. 14, 2531 (2002).

[26] J. J. Hopfield, Proc. Natl. Acad. Sci. USA 79, 2554 (1982).

[27] A. Sattar, S. Fostner, and S. A. Brown, Phys. Rev. Lett. 111, 136808 (2013).

[28] R. Landauer, IBM J. Res. Dev. 1, 223 (1957).

[29] D. A. Wharam, T. J. Thornton, R. Newbury, M. Pepper, H. Ahmed, J. E. F. Frost, D. G. Hasko, D. C. Peacock, D. A. Ritchie, and G. A. C. Jones, J. Phys. C 21, L209 (1988).

[30] B. J. van Wees, H. van Houten, C. W. J. Beenakker, J. G. Williamson, L. P. Kouwenhoven, D. van der Marel, and C. T. Foxon, Phys. Rev. Lett. 60, 848 (1988).

[31] D. Stauffer and A. Aharony, Introduction to Percolation Theory, 2nd ed. (CRC Press, New York, 1994).

[32] E. Nedaaee Oskoee and M. Sahimi, Phys. Rev. E 83, 031105 (2011).

[33] S. Fostner, R. Brown, J. Carr, and S. A. Brown, Phys. Rev. B 89, 075402 (2014).

[34] J. Schmelzer, S. A. Brown, A. Wurl, M. Hyslop, and R. J. Blaikie, Phys. Rev. Lett. 88, 226802 (2002).

[35] A. D. Dunbar, J. G. Partridge, M. Schulze, and S. A. Brown, Eur. Phys. J. D 39, 415 (2006).
[36] J. Quintanilla and S. Torquato, Phys. Rev. E 54, 5331 (1996).

[37] K. C. Chang and T. Odagaki, Phys. Rev. B 35, 2598 (1987).

[38] I. Balberg, Phys. Rev. Lett. 59, 1305 (1987).

[39] Y. Hara and T. Odagaki, J. Phys. Soc. Jpn. 69, 3315 (2000).

[40] G. Ambrosetti, I. Balberg, and C. Grimaldi, Phys. Rev. B 82, 134201 (2010).

[41] C. Grimaldi, Phys. Rev. B 89, 214201 (2014).

[42] E. T. Gawlinski and H. E. Stanley, J. Phys. A 14, L291 (1981).

[43] S. Feng, B. I. Halperin, and P. N. Sen, Phys. Rev. B 35, 197 (1987).

[44] It is well established that allowing the particle size to vary has no substantive effect on the percolating properties of the system [31]. Our preliminary simulations indicate that this is also true in the tunneling regime.

[45] Units are volts per cluster diameter.

[46] C. Untiedt, M. J. Caturla, M. R. Calvo, J. J. Palacios, R. C. Segers, and J. M. van Ruitenbeek, Phys. Rev. Lett. 98, 206801 (2007).

[47] T. Tsong, Phys. Rev. B 44, 13703 (1991).

[48] M. Olsen, M. Hummelgård, and H. Olin, PLoS ONE 7, e30106 (2012).

[49] B. I. Shklovski and A. L. Éfros, Sov. Phys. Usp. 18, 845 (1975).

[50] M. D. Pickett, G. Medeiros-Ribeiro, and R. S. Williams, Nat. Mater. 12, 114 (2012).

[51] B.-Y. Choi, S.-J. Kahng, S. Kim, H. Kim, H. W. Kim, Y. J. Song, J. Ihm, and Y. Kuk, Phys. Rev. Lett. 96, 156106 (2006).

[52] J. Mativetsky, G. Pace, M. Elbing, M. Rampi, M. Mayor, and P. Samorì, J. Am. Chem. Soc. 130, 9192 (2008).

[53] M. Min, S. Seo, S. M. Lee, and H. Lee, Adv. Mater. 25, 7045 (2013).

[54] J. W. Choi, A. H. Flood, D. W. Steuerman, S. Nygaard, A. B. Braunschweig, N. N. P. Moonen, B. W. Laursen, Y. Luo, E. Delonno, A. J. Peters, J. O. Jeppesen, K. Xu, J. F. Stoddart, and J. R. Heath, Chem. Eur. J. 12, 261 (2005).

[55] J. R. Heath, Annu. Rev. Mater. Res. 39, 1 (2009).

[56] C. Joachim, J. Gimzewski, and A. Aviram, Nature (London) 408, 541 (2000).

[57] J. Tour, W. Van Zandt, C. Husband, S. Husband, L. Wilson, P. Franzon, and D. Nackashi, IEEE Transactions On Nanotechnology 1, 100 (2002).

[58] C. Busche, L. Vilà-Nadal, J. Yan, H. N. Miras, D.-L. Long, V. P. Georgiev, A. Asenov, R. H. Pedersen, N. Gadegaard, M. M. Mirza, D. J. Paul, J. M. Poblet, and L. Cronin, Nature (London) 515, 545 (2014).

[59] A. Mazzoni, F. D. Broccard, E. Garcia-Perez, P. Bonifazi, M. E. Ruaro, and V. Torre, PLoS ONE 2, e439 (2007).

[60] V. Pasquale, P. Massobrio, L. L. Bologna, M. Chiappalone, and S. Martinoia, Neuroscience 153, 1354 (2008).

[61] A. Sasaki, Y. Kishimoto, E. Takahashi, S. Kato, T. Fujii, and S. Kanazawa, Phys. Rev. Lett. 105, 075004 (2010).

[62] S. Matsumoto and T. Odagaki, J. Phys. Soc. Jpn. 83, 034006 (2014). 